Running head: Mere exposure effect and selective attention

\title{
Attentional modulation of the mere exposure effect
}

Yoshihiko Yagi ${ }^{1}$, Shinobu Ikoma ${ }^{2}$, and Tadashi

$\mathrm{Kiku} \mathrm{chi}{ }^{2}$

${ }^{1} \mathrm{National}$ Institute of Advanced Industrial Science and Technology

${ }^{2}$ University of Tsukuba, Graduate School of Comprehensive Human Sciences

Word count: 7173

Corresponding author: Yoshihiko Yagi

Address: AIST Tsukuba Central 6, 1-1-1 Higashi, Tsukuba, Ibaraki $305-8566$, J a pan

E-mail:y-yagi@aist.go.jp

Phone: +81-29-861-6724 (lab.) 
Abstract $(133$ words $)$

The mere exposure effect refers to the phenomenon where previous exposures to stimuli increase participants, subsequent affective preference for those stimuli. This study explored the effect of selective attention on the mere exposure effect. The experiments manipulated the to-be-attended drawings in the exposure period (either red orgreen polygons in Experiments 1 and 2; both red and green polygons in Experiments 3 and 4) and black to-be-evaluated drawings in the affective judgment period (morphologically identical to the red or green polygons in Experiments 1 and 4; morphologically identical to the composite drawings in Experiments 2 and 3). The results showed a significant mere exposure effect only for the target shapes involved in attentional selection, even when the participants could recognize the non-target shapes. This indicates that selective attention modulated the mere exposure effect.

Key words: mere exposure effect, affect, selective a t tention 
Numerous attempts have been made to elucidate the process of intuitive emotional evaluations of unfamiliar objects. Since the seminal research of Zajonc (1968), this topic has been studied extensively in the context of the mere exposure effect, where repeated exposure to a previously novel stimulus causes an increase in positive affect toward that stimulus. In the more than 200 research articles on this topic (see, Monahan, Murphy, \& Zajonc, 2000, p. 462), few have investigated cognitive factors in the exposure effect, such as selective attention, level of processing, and cognitive load. Most studies have focused on stimulus presentation factors such as frequency, duration, type, or complexity of exposed stimuli (see Bornstein, 1989). Researchers have generally presumed that repeated exposure to a novel stimulus would lead to positive evaluation of that stimulus with minimal intervening cognitive activity (e.g., Monahan et al., 2000; Winkielman \& Cacioppo, 2001). This is consistent with ample evidence that the mere exposure effect could be seen even when participants could not discern the exposed stimuli due to brief or masked presentation (Bornstein \& D'Agostino, 1992; Elliott \& Dolan, 1998; Kunst-Wilson \& Zajonc, 1980; Mandler, Nakamura, \& Van Zandt, 1987; Monahan et al., 2000). However, a recent study showed that the mere exposure effect for an ambiguous duck/rabbit figure was influenced by instructions concerning how to interpret the figure (i.e., as a duck or rabbit) during the exposure period, which suggests that higher cognitive functions are involved in the mere exposure effect (Craver-Lemley \& Bornstein, 2006).

Given that cognitive processes could modulate the mere exposure 
effect, one plausible influence is selective attention. Attending or ignoring a stimulus results in different states of memory representation for that stimulus, as demonstrated in numerous studies (e.g., Neill, 1977; Rock \& Gutman, 1981; Tipper, 1985) as well as neuroimaging findings (e.g., Vuilleumier, Schwartz, Duhoux, Dolan, \& Driver, 2005). For example, Tipper (1985) showed that previously to-be-attended or to-be-ignored items resulted in relatively faster (i.e., repetition priming) or slower (i.e., negative priming) responses to those stimuli in subsequent trials. This indicates that both the to-be-attended and to-be-ignored items were identified, and then facilitated or inhibited responses. Since the mere exposure effect involves memory of the previously exposed stimuli, it is plausible that differences in the memory state of the stimuli could modulate the effect. Also, there is increasing evidence indicating a reciprocal influence of attention on emotion. Raymond, Fenske and their colleagues reported a series of experiments in which a simple visual search task and an affective evaluation task were serially combined in a trial (Fenske, Raymond, Kessler, Westoby, \& Tipper, 2005; Fenske, Raymond, \& Kunar, 2004; Raymond, Fenske, \& Tavassoli, 2003; Raymond, Fenske, \& Westoby, 2005; for a review, see Fenske \& Raymond, 2006). In the preceding visual search task, participants were asked to localize or identify a target while ignoring other distractor(s). Immediately after their response for the visual search task, participants were presented with a stimulus from one of the three stimulus-categories (i.e., previous target, previous distractor, or novel stimulus) and asked to affectively evaluate it. The results consistently showed that the previous distractors 
were evaluated more negatively than the previous targets or novel stimuli (distractor devaluation effect; see also Goolsby, Shapiro, \& Raymond, 2009; Goolsby, Shapiro, Silvert, et al., 2009; Griffiths \& Mitchell, 2007; Kiss, et al., 2007; Veling, Holland, \& Knippenberg, 2007). These findings clearly revealed that attention could modulate the affective evaluation. However, they do not provide direct evidence for attentional modulation of the mere exposure effect, because they showed devaluation for the ignored stimuli but not an increase in positive affect.

The purpose of our study is to investigate whether attentional selection modulates the mere exposure effect. We conducted four mere exposure experiments. In the exposure phase, composite drawings, composed of a red and a green nonsense line-drawing (polygon), were presented. Participants were asked to focus their attention on either or both of the colored components, and to memorize them. In the affective judgment phase, they were presented with pairs of an exposed and a novel stimulus in black, and asked to choose the one that they like more. To-be-attended drawings in the exposure phase (either red or green polygons in Experiments 1 and 2; both red and green polygons in Experiments 3 and 4) and to-be-evaluated drawings in the affective judgment phase (morphologically identical to the red or green polygons in Experiments 1 and 4; morphologically identical to the composite drawings in Experiments 2 and 3) were manipulated across the experiments (see Figure 1). 
Insert Figure 1 about here

It should be noted that prior to the information processing stages for identification, the processing of the to-be-ignored or unattended stimuli would not be terminated (see, Lavie, 1995, 2000; Lavie \& Fox, 2000). Using similar composite drawings as stimuli, DeSchepper and Treisman (1996) showed robust negative priming for the to-be-ignored components, which indicates that a substantial memory trace even of the ignored stimuli could be constructed in this paradigm. Both the to-be-attended and the to-be-ignored items would be perceived. However, subsequent to the information processing stages for identification, the former would be facilitated and the latter would be inhibited. Therefore, attentional modulation of the mere exposure effect would clarify whether the exposure effect would occur for any perceived stimuli that are presented with an adequate physical property (i.e., eccentricity, duration, and luminance). Moreover, a demonstration of attentional modulation could be a first step to open discussion about the locus of the increase in positive affect toward previously novel stimuli through the information processing system (Craver-Lemley \& Bornstein, 2006).

If selective attention could modulate the mere exposure effect, then the effect should be seen only toward the items that are the target of attentional selection. According to the perceptual fluency hypothesis, one widely accepted view, the mere exposure effect results from enhanced perceptual fluency (i.e., the ease of perceptual processing) due to prior 
experience and misattribution of that fluency as liking for the previously exposed stimuli (Bornstein \& D’Agostino, 1994; Jacoby, Kelley, \& Dywan, 1989; Whittlesea, 1993; Whittlesea \& Price, 2001). Based on this view, the affective evaluations of the to-be-attended drawings should be higher than those of the to-be-ignored or novel drawings, because it is well know that attending to a specific stimulus facilitates subsequent perceptual processing of that stimulus, relative to a novel or previously ignored stimulus. On the other hand, if selective attention has little to do with the mere exposure effect, then the same degree of the effect would be expected across the all the experiments, because the exposed stimuli are identical among all the experiments. 


\section{Experiment 1}

The purpose of Experiment 1 is to demonstrate attentional modulation of the mere exposure effect. The experiment is composed of three phases: the exposure phase, the affective judgment phase, and the recognition phase. In the exposure phase, participants are presented with composite drawings, each composed of a red and a green polygon, and asked to focus their attention on one polygon, while ignoring the other. Each composite drawing was consistently composed of the same pair of two polygons, so the exposure frequencies of the attended and ignored stimuli are identical. If an increase in positive affect toward exposed stimuli simply depends on exposure frequency, the same degree of the mere exposure effect would be expected for attended and ignored stimuli.

\section{Method}

Participants. Thirty-two (13 men and 19 women) undergraduate or graduate students (mean age: 20.46, SD: 1.27) participated in this experiment. All of them had normal or corrected-to-normal vision and did not know the purpose of the experiment.

Apparatus. This experiment was controlled by a Pentium-IV computer running Microsoft Windows XP. Stimuli were displayed on a color monitor and participants responded using a response pad (Cedrus, RB-520) connected to the computer. The participants sat in a dark room with their heads fixed in a headrest. The distance between the headrest 
and the computer monitor was approximately $57.3 \mathrm{~cm}$.

Stimuli. We generated 90 line-drawings of polygons, using Vanderplas and Garvin (1959) as a model. The polygons had 4, 6, 8, or 12 corners; the size of the largest polygon was $7^{\circ} \times 7^{\circ}$, and the smallest was $5^{\circ} \times 2.3^{\circ}$. We also generated 45 composite drawings by overlapping two polygons. Some of these yoked polygons had the same number of corners and others did not. Five of the 45 composite drawings were used as filler stimuli to prevent primacy and recency effects. In the exposure phase, the composite drawings were used as the exposed stimuli. One of the component polygons in the composite drawings was drawn in red lines $\left(10.67 \mathrm{~cd} / \mathrm{m}^{2}\right)$ and the other was green $\left(3.07 \mathrm{~cd} / \mathrm{m}^{2}\right)$. In the affective judgment phase and the recognition phase, black $\left(0.02 \mathrm{~cd} / \mathrm{m}^{2}\right)$ polygons morphologically identical to the components in the composite drawings were presented. As a fixation point, a black “+” symbol $\left(0.7^{\circ} \times 0.7^{\circ}\right)$ was utilized. All stimuli were presented on a white background $\left(36.57 \mathrm{~cd} / \mathrm{m}^{2}\right)$.

Procedure. To eliminate memory biases in the affective judgment phase, such as explicit selection of the exposed stimuli, the participants were instructed that the purpose of the experiment was to investigate the relation between color-based attentional selection and memory performance. The true purpose, to investigate affective judgments, was explained in debriefing at the end of the experiment.

In the exposure phase, 20 composite drawings were randomly chosen as the exposed stimuli. Each trial began with the appearance of a fixation cross for $1 \mathrm{~s}$. Then a colored composite drawing was presented at the center of the display for $1 \mathrm{~s}$. After a $2 \mathrm{~s}$ blank interval, the fixation for the 
next trial automatically appeared. The participants were asked to focus their attention on one of the two components in the composite drawings (to-be-attended polygons) while ignoring the other (to-be-ignored polygons), and to memorize as many of the attended polygons as possible. The red polygons were assigned as the to-be-attended stimuli for half of the participants and the green polygons were assigned as the to-be-attended stimuli for the remaining half. Each composite drawing was presented three times in random order, with the constraint that the same drawing was never presented three times in succession. In the first and last five trials, 5 filler composite drawings were presented, each individually twice. Thus, the participants observed a total of 70 presentations. All stimulus properties (exposed or novel, location, color) were counterbalanced between participants.

At the beginning of the affective judgment phase, participants were told that this phase had no relation to the memory test and would be conducted just for collecting preliminary data. Each trial began with the appearance of a fixation cross for $1 \mathrm{~s}$. Then, a pair of black polygons was presented, one at each side of the fixation. One of the two polygons was morphologically identical to the to-be-attended or to-be-ignored component polygons in the exposed composite drawings. The other was a novel polygon that had the same number of corners as its yoke. The participants were asked to choose the one that they liked more. They reported their preference for the left (or right) drawing by pressing the left (or right) button on the response pad. After a $2 \mathrm{~s}$ blank interval, a fixation for the next trial automatically appeared. Forty pairs of an 
exposed polygon (20 attended and 20 ignored) and a novel polygon were presented, once per pair, and no practice trial was conducted.

At the beginning of the recognition phase, the participants were told that the purpose of this experiment was to test memory performance not only for the to-be-attended stimuli, but also for the to-be-ignored stimuli. Thus, they were encouraged to try to recognize both the to-be-attended and the to-be-ignored stimuli. The procedure of this phase was identical to that of the affective judgment phase, with the following exception. A black polygon was presented at the center of the display that was either novel or morphologically identical to a to-be-attended or to-be-ignored polygon. We gave the participants a two-alternative forced-choice task asking them to discriminate whether the polygon had been presented in the exposure phase, irrespective of whether it was to-be-attended or to-be-ignored. If they thought that it had been (or had not been) previously presented, half of them responded by pressing the left (or right) button; the responses were reversed for the remaining half of the participants. Eighty black polygons (20 attended, 20 ignored, and 40 novel stimuli) were presented, each individually once.

\section{Results and Discussion}

Table 1 shows the mean selection rates for the exposed drawings in the affective judgment phase and the mean proportions of correct responses in the recognition phase, as a function of attentional state (to-be-attended or to-be-ignored). 
Insert Table 1 about here

The proportion of correct recognition for the to-be-attended polygon was calculated from the hit (reporting old when the stimulus was old) rate for the to-be-attended polygons and the correct recognition (reporting new when the stimulus was new) rate for the novel polygons for each participant. The equivalent calculation was done for the to-be-ignored polygons. Two one-sample $t$-tests showed that the proportion of correct recognition for the to-be-attended polygon was higher than the .50 chance level $(t(31)=6.54, p<.001)$, and that there was no significant difference between the proportion for the to-be-ignored polygon and the .50 chance level $(t(31)=1.05, p=.30)$. A two $t$-test for these values revealed that recognition performance for the to-be-attended polygons was significantly higher than that for the to-be-ignored polygons $(t(31)=5.08, p<.01)$. These indicate that the participants were attending to the appropriate colored components in the composite drawings during the exposure period.

The selection rate for the exposed drawings indicates the proportion of trials where participants chose an exposed polygon as preferable in the affective judgment phase. To assess the occurrence of the mere exposure effect, one-sample $t$-tests were conducted for the mean selection rates for the to-be-attended and to-be-ignored stimuli. The results show that the to-be-attended polygon was chosen as affectively preferable significantly 
more often than the .50 chance level $(t(31)=2.77, p<.01)$, but the to-be-ignored polygon was not affectively preferable more than chance $(t(31)=-0.59, p=.55)$. A two-sample $t$-test revealed that the mean selection rate for the to-be-attended polygon was higher than for the to-be-ignored polygon $(t(31)=2.29, p<.05)$. These results indicate that the to-be-attended polygons were evaluated as more likable than the to-be-ignored and the novel stimuli. Thus, the results of Experiment 1 demonstrate attentional modulation of the mere exposure effect. The to-be-ignored stimuli were evaluated equally favorably as the novel stimuli. Thus we did not find evidence that inhibitory processes of attention devaluated the ignored stimuli compared to the novel stimuli.

\section{Experiment 2}

In Experiment 2, participants were asked to focus their attention on one of the two components in the composite drawings while ignoring the other in the exposure phase, and to evaluate the black composite drawings in the affective judgment phase. The black composite drawings were morphologically identical to the images projected to the retina in the exposure phase. If any retinal images would induce an increase in positive affect independently of attentional selection, then the same degree of the mere exposure effect as for the to-be-attended polygons in Experiment 1 would be seen. On the other hand, if attentional selection inhibits an increase in positive affect toward the images that are not the target of attention, then no mere exposure effect would be seen. 


\section{Method}

Participants. Thirty-two (21 men, 11 women) undergraduate or graduate students (mean age: 20.71, SD: 1.92) participated in the experiment; none had participated in Experiment 1. All of them had normal or corrected-to-normal vision and did not know the purpose of the experiment.

Apparatus and Stimuli. The apparatus and stimuli utilized in this experiment were the same as in Experiment 1.

Procedure. The procedures utilized in this experiment were the same as those in Experiment 1 except for the following. In the affective judgment phase, 20 pairs of black composite drawings were presented. One member of each pair was morphologically identical to the colored composite drawings, while the other was a novel stimulus that had the same number of corners as the yoke.

\section{Results and Discussion}

The results of this experiment are summarized in Table 1. Two one-sample $t$-tests showed that the proportion of correct recognition was higher than the .50 chance level, not only for the to-be-attended polygon $(t(31)=9.99, p<.001)$, but also for the to-be-ignored polygon $(t(31)=2.08$, $p<.05)$. Slightly better recognition performance for the to-be-ignored polygon in this experiment relative to that in Experiment 1 might be attributed to the smaller proactive interference. Participants in Experiment 1 were presented with the exact same drawings between the 
affective judgment phase and the recognition phase. This was not the case in this experiment. At any rate, a two sample $t$-test revealed that the proportion of correct recognition for the attended polygons was significantly higher than for the ignored polygons $(t(31)=8.81, p<.001)$. As in Experiment 1, this result indicates that the participants actually attended to the designated colored drawings.

To assess the occurrence of the mere exposure effect, a one-sample $t$-test was conducted for the mean selection rate as preferable in the affective judgment phase for the exposed drawings. There was no significant difference between this proportion and the .50 chance level $(t$ $(31)=-0.46, p=.64)$, despite the fact that the stimuli used in the exposure phase of this experiment were the same as those in Experiment 1. Moreover the contours of the black composite drawings presented in the affective judgment phase were morphologically identical to the retinal images of the exposed drawings in this experiment. These results indicate that the occurrence of the mere exposure effect is not dependent on the images projected to retina, but on the shapes of the attended stimuli.

However, another explanation may account for these findings. The results could be attributed to the dissimilarity between the drawings in the exposure phase and those in affective judgment phase. The colored and black composite drawings shared their overall outline shapes, but the latter had lost some information which the former had. This may have caused the participants to explicitly or implicitly perceive that the previously exposed drawings and the to-be-evaluated drawings were not identical. We test this possibility in the next experiment. 


\section{Experiment 3}

The results of Experiment 2 might be attributed to the dissimilarity of the stimuli between the exposure and affective judgment phases. If this is the case, then the same results should be found even when participants are asked to focus their attention on the whole shapes of a colored composite drawing (i.e., on both the red and green polygons as a whole) in the exposure phase. On the other hand, from the perspective that an increase in positive affect would occur only for the target of attentional selection, the mere exposure effect should be found in this situation. In Experiment 3 , we present the same stimuli from Experiment 2 in order to test these two possibilities.

\section{Method}

Participants. Thirty-two (16 men, 16 women) undergraduate or graduate students (mean age: 20.62, SD: 1.57) participated in the experiment; none had participated in the previous experiments. All of them had normal or corrected-to-normal vision and did not know the purpose of the experiment.

Apparatus and Stimuli. The apparatus and stimuli utilized in this experiment were the same as in Experiment 1.

Procedure. The procedure utilized in this experiment was the same as in Experiment 2 with the following changes. In the exposure phase of this experiment, the participants were asked to focus their attention on 
both the colored polygons as one composite drawing and to memorize as many of the composite drawings as possible. In the affective judgment phase, similar to Experiment 2, the stimuli were 20 pairs of exposed composite drawings and novel composite drawings. In the recognition phase, 40 (20 exposed and 20 novel) composite drawings were presented, each individually once.

\section{Results and Discussion}

The results of this experiment are summarized in Table 1. A one-sample $t$-test revealed that the proportion for correct recognition in this experiment was higher than the .50 chance level $(t(31)=5.22, p$ $<.001)$. This indicates that the participants actually focused their attention on both components of the colored composite drawings and constructed integrated perceptual representations.

A one-sample $t$-test revealed that there was a significant difference between the mean selection rate as preferable in the affective judgment phase for the exposed drawings and the .50 chance level $(t(31)=3.37, p$ $<.01)$. Thus the mere exposure effect was obtained in this experiment, despite the fact that the stimuli used in Experiment 2 and Experiment 3 were identical. This result suggests that the lack of the mere exposure effect in Experiment 2 could not be attributed to the dissimilarity of the stimuli between the exposure phase and the affective judgment phase.

\section{Experiment $4 a$}


The findings from the previous three experiments consistently indicated attentional modulation of the mere exposure effect. The mere exposure effect was seen only for the target shape of attentional selection. The remaining question concerns the exposure effect for the components of the attended composite drawings. They are neither the target shapes of attentional selection nor to-be-ignored, but are exposed components of the attended composite drawings. Experiment 4 a tested the mere exposure effect for the components of the attended composites. We presented the same stimuli as in Experiment 1 in the exposure phase and the affective judgment phase. The participants were asked to focus their attention on both the red and green components in the composite drawings and encouraged to observe the two colored-components as one unified drawing in the exposure phase. Then they were asked to evaluate black polygons that were morphologically identical to the components in the exposed composite drawings.

\section{Method}

Participants. Thirty-two (8 men, 24 women) undergraduate or graduate students (mean age: 20.28, SD: 1.97) participated in this experiment; none had participated in the previous experiments. All of them had normal or corrected-to-normal vision and did not know the purpose of the experiment.

Apparatus and Stimuli. The apparatus and stimuli utilized in this experiment were the same as in Experiment 1.

Procedure. The procedure utilized in this experiment was the same 
as that used in Experiment 3, except that the participants were asked to focus their attention on both the colored polygons as one unified drawing in the exposure phase, and that 40 pairs of exposed and novel component polygons were presented in the affective judgment phase.

\section{Results and Discussion}

The results of this experiment are summarized in Table 1. A one-sample $t$-test revealed that the proportion for correct recognition in this experiment was higher than the .50 chance level $(t(31)=5.56, p$ $<.001)$. This indicates that the participants actually focused their attention on both components of the colored composite drawings and constructed integrated perceptual representations.

A one-sample $t$-test revealed that there was no significant difference between the mean selection rate as preferable in the affective judgment phase for exposed drawings and the .50 chance level $(t(31)=-0.38, p$ $=.70)$. This indicates that no mere exposure effect was found in this experiment. Both the red and green polygons were components of the attended composite drawings, but were not the to-be-attended shape itself. Therefore, the findings of this experiment demonstrate a reduction of the mere exposure effect for the components of the attended composite drawings. This indicates that the mere exposure effect would occur only for the items that are exactly attended to, but not for its components.

\section{Experiment $4 b$}


The finding from Experiment $4 \mathrm{a}$ indicates that the mere exposure effect would be seen only for the exact items that participants attended to. The remaining question concerns the role of selective attention in the reduction of the mere exposure effect toward the non-target stimuli of attentional selection. There seem to be two possibilities for the role of attention. One is that attending to the composite drawings would restrict the accessibility in memory of any non-target stimuli of attentional selection, even when those stimuli are components in the attended composites. This restriction might also reduce the exposure effect. The other possibility is that attentional selection would inhibit increasing positive affect, independently of memory accessibility.

Experiment $4 \mathrm{~b}$ was designed to distinguish between these two alternatives. The procedures of the exposure and affective judgment phases were identical to those in Experiment 4 a. In the recognition phase, however, the participants were presented with the components, not the composite drawings, and the recognition task was unexpected.

\section{Method}

Participants. Thirty-two (14 men, 18 women) undergraduate or graduate students (mean age: 20.31, SD: 1.37) participated in this experiment; none had participated in the previous experiments. All of them had normal or corrected-to-normal vision and did not know the purpose of the experiment.

Apparatus and Stimuli. The apparatus and stimuli utilized in this experiment were the same as in Experiment 1. 
Procedure. The procedure utilized in this experiment was the same as that used in Experiment $4 \mathrm{a}$ with the following changes. In the recognition phase, similar to Experiment 1, 80 black component drawings (40 exposed and 40 novel stimuli) were presented, each individually once. Participants were asked to report whether the drawings had been presented in the exposure phase. Note that the participants had been asked to memorize the shapes of the composite drawings at the beginning of the experiment.

\section{Results and Discussion}

The results of this experiment are summarized in Table 1. A one-sample $t$-test revealed that there was no significant difference between the mean selection rate as preferable in the affective judgment phase for exposed drawings and the .50 chance level $(t(31)=0.99, \mathrm{p}=.32)$. This indicates that no mere exposure effect was found in this experiment.

A one-sample $t$-test showed that the proportion for correct recognition in this experiment was higher than the .50 chance level $(t(31)=3.56, p$ $<.001)$. This indicates that both components in the attended composite drawings were present in the accessible memory trace. Therefore attentional modulation of the mere exposure effect seems to occur, independently of the memory accessibility. Note that the results from the recognition task of this experiment are not conflicting with those for the ignored polygons in Experiment 1. The targets of the recognition task in Experiment $4 \mathrm{~b}$ were the components in the to-be-attended composite drawings. In contrast, the ignored polygons in Experiments 1 were 
distracting stimuli which potentially interfered with discerning the to-be-attended drawings, and thus they were to be actively inhibited.

\section{General Discussion}

The purpose of this study was to investigate whether attentional selection could modulate the mere exposure effect. We conducted four mere exposure experiments. In Experiment 1, the to-be-attended stimuli in the exposure phase were red or green polygons in a composite drawing and the to-be-evaluated stimuli in the affective judgment phase were black polygons. A significant mere exposure effect was found for the attended polygon stimuli. No evidence was found that inhibitory processes of attention devaluated the ignored stimuli lower than the novel stimuli. In Experiment 2, where the to-be-attended stimuli were the component polygons and the to-be-evaluated stimuli were the composite drawings, no mere exposure effect was found. In Experiment 3, where the composite drawings were the to-be-attended and to-be-evaluated stimuli, a significant mere exposure effect was found. In Experiment 4, the to-be-attended stimuli were the composite drawings and the to-be-evaluated stimuli were the polygon components. The results showed that the memory traces for the components in the attended composite drawings were available for the recognition judgment, but did not influence the affective judgment.

On the basis of these findings, we concluded that selective attention can modulate the mere exposure effect. The mere exposure effect would 
occur only for the representations formed via attentional selection, at least under circumstances where selective attention was involved in visual information processing. In other words, the mere exposure effect would be diminished for any images excluded from attentional selection, even if they were available for explicit memory retrieval.

This view of attentional modulation is also supported by a comparison across experiments. If attentional selection played a critical role in the occurrence of the mere exposure effect, the mean selection rate for exposed drawings should be higher in the situation where the shape of to-be-attended drawings and that of to-be-evaluated drawings were congruent than in the situation where those were incongruent. Experiment 3 and the attended stimuli of Experiment 1 were congruent situations. Experiment 2 and Experiment 4 were incongruent situations. In order to test the congruency effect, the data from 3 experiments (i.e., Experiments 2, 3, and 4a) and 1 experimental condition (i.e., the attended stimuli of Experiment 1) was combined into an exploratory ANOVA of the mean selection rates for exposed drawings with two between-group factors (to-be-attended drawings: components vs. composites; to-be-evaluated drawings: components vs. composites). The results of the ANOVA showed no significant main effects $\left(F_{\mathrm{S}}<1\right)$, but a significant interaction of the two factors $(F(1,124)=14.27, p<.001)$. This indicates that participants more frequently chose the exposed drawings as affectively preferable in the congruent situations than in the incongruent situations. This result is consistent with Craver-Lemley and Bornstein (2006) who suggested that higher cognitive processes are involved in the 
mere exposure effect. The present study extended their study and identified selective attention as one such cognitive process.

Another explanation which may account for the present findings is a recognition memory bias. According to this type of explanation, the participants simply chose the stimuli that they memorized as affectively preferable. If this were the case, then individuals who memorized more shapes of drawings should have a tendency to choose the exposed drawings more often in the affective judgment phase. Alternatively, specific items that more participants memorized should be chosen as preferable more often. We tested these possibilities with the data from the attended conditions of Experiment 1 and from Experiment 3, where significant mere exposure effects were found. Neither the individual participant nor the stimulus analysis showed a significant correlation between the proportion correct in the recognition phase and the mean selection rate for the exposed drawings in the affective judgment phase (for individual, $r(62)=.03, n_{s}$; for stimulus, $\left.r(118)=.101, n_{s}\right)$. These results are consistent with previous findings which showed no correlation between the mere exposure effect and recognition performance (e.g., Anand \& Sternthal, 1991; Matlin, 1971; Moreland \& Zajonc, 1977; Seamon, et al., 1995; Wilson, 1979). Moreover, in Experiment 4b, no mere exposure effect was found despite the fact that the proportion for correct recognition was significantly higher than chance level. Therefore, the present findings should not be attributed to simple response bias, but to attentional modulation.

The present findings also indicate that attentional modulation has 
two concrete roles in the exposure effect: one toward the ignored items and the other toward the attended items. For ignored items, attentional selection would reduce the positive affect toward the non-target stimuli, as discussed above. On the other hand, for attended items, attentional selection is likely to further increase positive affect for the target stimuli. Shimojo and his colleagues have recently reported that preference ratings for simple geometric figures were scarcely affected by repeated exposure of those figures (Liao \& Shimojo, 2008; Shimojo, Park, Lebon, Schleim, \& Shimojo, 2007; Shimojo, Park, Kashino, \& Shimojo, 2008). Our pilot study, where the same polygons as those used in this study were individually presented in the exposure phase, also showed no mere exposure effect. The findings from our pilot study and the previous studies reported by Shimojo and his colleagues indicate that repeated exposure of nonsense line-drawings would induce less positive affect in a supraliminal exposure situation (see also Bornstein, Kale, \& Cornell, 1990). However, nonsense line-drawings seem to be suitable for a subliminal mere exposure effect (e.g., Bornstein \& D’Agostino, 1992; Kunst-Wilson \& Zajonc, 1980). Despite the fact that no or little mere exposure effects could be seen for the nonsense line-drawings in the supraliminal exposure situation, the results for the attended polygons in Experiment 1 and from Experiment 3 showed significant mere exposure effects. Therefore it is reasonable to assume that a situation requiring attentional selection would enhance the mere exposure effect for the attended items, relative to a situation where attentional selection is not required. In short, we assume that attentional selection would modulate the mere exposure 
effect by reducing the visual representations constructed from one visual object. Therefore the increased positive affect would not be diffused within similar representations other than the exact same shape as the previously exposed one.

In our study, we found no evidence that the to-be-ignored items were devaluated. On the other hand, converging evidence suggests that the to-be-ignored items in a previous visual search task were devaluated (e.g., Raymond et al., 2003). Goolsby and his colleagues give one possibility to account for this discrepancy. According to Goolsby, Shapiro, Silvert, et al. (2009), the distractor devaluation is feature-based. For instance, if the target and distractor defining features are red and blue color respectively in the preceding visual search task, any stimuli with blue color would be devaluated in the subsequent affective judgment task. In addition. Goolsby, Shapiro, Silvert, et al. (2009) found that working memory load clearly reduced the amount of the distractor devaluation effect, indicating that distractor devaluation would require visual working memory resources. In the present study, the target and distractor defining features (i.e., color) were removed in the affective judgment phase and the task in the exposure phase included high visual working memory load.

It is beyond the scope of this study to examine the previous hypotheses for the mere exposure effect. However, it is worthwhile to discuss the consistency between the present findings and the previous hypotheses. One of the prevailing hypotheses is the perceptual fluency hypothesis (e.g., Bornstein \& D'Agostino, 1994). As described in the 
Introduction, it is uncontroversial that attending to specific items would facilitate subsequent information processing involving those items. Therefore, the present findings are consistent with the perceptual fluency hypothesis, assuming that the terminology of "perceptual" includes high-level cognitive processes such as constructing perceptual representations after attentional selection (Willems \& Van der Linden, 2006). Another hypothesis postulates that repeated exposure reduces alertness and tension for novel stimuli, which causes positive evaluations of exposed stimuli (Monahan et al., 2000). This view is also consistent with the findings of our study, given that attending to specific items enhances the efficiency of learning about their harmlessness. Shimojo and his colleagues recently proposed a new hypothesis that active information processing, such as orienting behavior, itself contributes to increasing the attractiveness of the target stimuli (Shimojo, Simion, Shimojo, \& Scheier, 2003; Simion \& Shimojo, 2006). The findings of our study are also compatible with this view, because it seems rational to regard the processes of attentional selection as one form of active information processing.

The present findings clearly demonstrate a contribution of selective attention to the mere exposure effect. However, there are some unsolved issues. For instance, it is not still clear that how attentional selection can affect the subliminal mere exposure effect, even though we suppose that participants were not unattending to the spatial location where the stimuli were presented in the subliminal exposure studies. Moreover, although our demonstration is the first step in opening discussion about 
the locus of the increase in positive affect toward exposed stimuli, there remain two possibilities about the role of selective attention in the mere exposure effect. One possibility is that the increase in positive affect occurs after attentional selection. The other possibility is that attentional selection modulates positive affect, after exposed stimuli elicited positive affect. Thus, facilitatory mechanisms might further increase positive affect for the target stimuli of selection, and inhibitory mechanisms might inhibit that affect for non-target stimuli. Further studies are needed to clarify these issues. 


\section{References}

Anand, P., \& Sternthal, B. (1991). Perceptual fluency and affect without cognition. Memory \& Cognition, 19, 293-300.

Bornstein, R. F. (1989). Exposure and affect: Overview and meta-analysis of research, 1967-1987. Psychological Bulletin, 106, 265-289.

Bornstein, R. F., \& D'Agostino, P. R. (1992). Stimulus recognition and the mere exposure effect. Journal of Personality and Social Psychology, $63,545-552$.

Bornstein, R. F., \& D'Agostino, P. R. (1994). The attribution and discounting of perceptual fluency: Preliminary tests of a perceptual fluency/attributional model of the mere exposure effect. Social Cognition, 12, 103-128.

Bornstein, R. F., Kale, A. R., \& Cornell, K. R. (1990). Boredom as a limiting condition on the mere exposure effect. Journal of Personality and Social Psychology, 58, 791-800.

Craver-Lemley, C., \& Bornstein, R. F. (2006). Self-generated visual imagery alters the mere exposure effect. Psychonomic Bulletin \& Review, 13, 1056-1060.

DeSchepper, B., \& Treisman, A. (1996). Visual memory for novel shapes: Implicit coding without attention. Journal of Experimental Psychology: Learning, Memory, and Cognition, 22, 27-47.

Elliott, R., \& Dolan, R. J. (1998). Neural response during preference and memory judgments for subliminally presented stimuli: A functional neuroimaging study. The Journal of Neuroscience, 18, 4697-4704. 
Fenske, M. J., Raymond, J. E., Kessler, K., Westoby, N., \& Tipper, S. P. (2005). Attentional inhibition has social-emotional consequences for unfamiliar faces. Psychological Science, 16, 753-758.

Fenske, M. J., \& Raymond, J. E. (2006). Affective influence of selective attention. Current Directions in Psychological Science, 15, 312-316.

Fenske, M. J., Raymond, J. E., \& Kunar, M. A. (2004). The affective consequences of visual attention in preview search. Psychonomic Bulletin \& Review, 11, 1055-1061.

Goolsby, B. A., Shapiro, K. L., \& Raymond, J. E. (2009). Distractor devaluation requires visual working memory. Psychonomic Bulletin \& Review, 16, 133-138.

Goolsby, B. A., Shapiro, K. L., Silvert, L., Kiss, M., Fragopanagos, N., Taylor, J. G., Eimer, M., Nobre, A. C., \& Raymond, J. E. (2009). Feature-based inhibition underlies the affective consequences of attention. Visual Cognition, 17, 500-530.

Griffiths, O., \& Mitchell, C. J. (2007). Negative priming reduces affective ratings. Cognition \& Emotion, 22, 1119-1129.

Halpern, A. R., \& O’Connor, M. G. (2000). Implicit memory for music in Alzheimer's disease. Neuropsychology, 14, 391-397.

Jacoby, L. L., Kelley, C. M., \& Dywan, J. (1989). Memory attributions. In H. L. Roediger \& F. I. M. Craik (Eds.), Varieties of memory and consciousness: Essays in honour of Endel Tulving (pp. 391-422). Hillsdale, NJ: Erlbaum.

Kiss, M., Goolsby, B. A., Raymond, J. E., Shapiro, K. L., Silvert, L., Nobre, A. C., Fragopanagos, N., Taylor, J. G., \& Eimer, M. (2007). Efficient 
attentional selection predicts distractor devaluation: Event-related potential evidence for a direct link between attention and emotion. Journal of Cognitive Neuroscience, 19, 1316-1322.

Kunst-Wilson, W. R., \& Zajonc, R. B. (1980). Affective discrimination of stimuli that cannot be recognized, Science, 207, 557-558.

Lavie, N. (1995). Perceptual load as a necessary condition for selective attention. Journal of Experimental Psychology: Human Perception and Performance, 21, 451-468.

Lavie, N. (2000). Selective attention and cognitive control: Dissociating attentional function through different type of load. In S. Monsell \& J. Driver (Eds.), Control of cognitive processes: Attention and performance XVIII (pp. 175-194). Cambridge, MA: MIT Press.

Lavie, N., \& Fox, E. (2000). The role of perceptual load in negative priming. Journal of Experimental Psychology: Human Perception and Performance, 26, 1038-1052.

Liao, H.-I., \& Shimojo, S. (2008). Novelty vs. familiarity principles in preference decision: Task-context of memory matters [Abstract]. Journal of Vision, 8, 522a, from http://journalofvision.org/8/6/522/

Mandler, G., Nakamura, Y., \& Van Zandt, B. J. S. (1987). Nonspecific effects of exposure on stimuli that cannot be recognized. Journal of Experimental Psychology: Learning, Memory, and Cognition, 13, 646-648.

Matlin, M. W. (1971). Response competition, recognition, and affect. Journal of Personality and Social Psychology, 19, 295-300.

Monahan, J. L., Murphy, S. T., \& Zajonc, R. B. (2000). Subliminal mere 
exposure: Specific, general, and diffuse effects. Psychological Science, $11,462-466$.

Moreland, R. L., \& Zajonc, R. B. (1977). Is stimulus recognition a necessary condition for the occurrence of exposure effects? Journal of Personality and Social Psychology, 35, 191-199.

Neill, W. T. (1977). Inhibitory and facilitatory processes in selective attention. Journal of Experimental Psychology: Human Perception and Performance, 3, 444-450.

Raymond, J. E., Fenske, M. J., \& Tavassoli, N. (2003). Selective attention determines emotional responses to novel visual stimuli. Psychological Science, 14, 537-542.

Raymond, J. E., Fenske, M. J., \& Westoby, N. (2005). Emotional devaluation of distracting patterns and faces: A consequence of attentional inhibition during visual search? Journal of Experimental Psychology: Human Perception and Performance, 31, 1404-1415.

Rock, I., \& Gutman, D. (1981). The effect of inattention and form perception. Journal of Experimental Psychology: Human Perception and Performance, 7, 275-285.

Seamon, J. G., Williams, P. C., Crowley, M. J., Kim, I. J., Langer, S. A., Orne, P. J., \& Wishengrad, D. L. (1995). The mere exposure effect is based on implicit memory: Effects of stimulus type, encoding conditions, and number of exposures on recognition and affect judgments. Journal of Experimental Psychology: Learning, Memory, and Cognition, 21, 711-721.

Shimojo, S., Simion, C., Shimojo, E., \& Scheier, C. (2003). Gaze bias both 
reflects and influences preference. Nature Neuroscience, 6 , $1317-1322$.

Shimojo, E., Park, J., Kashino, M., \& Shimojo, S. (2008). Familiarity for faces and novelty for natural scenes in preference: Does similarity matter? [Abstract]. Journal of Vision, 8, 1137a, from http://journalofvision.org/8/6/1137/

Shimojo, E., Park, .J., Lebon, L., Schleim, S., \& Shimojo, S. (2007). Familiarity vs. novelty principles for preference [Abstract]. Journal of Vision, 7, 933a, from http://journalofvision.org/7/9/933/

Simion, C., \& Shimojo, S. (2006). Early interactions between orienting, visual sampling and decision making in facial preference. Vision Research, 46, 3331-3335.

Tipper, S. P. (1985). The negative priming effect: Inhibitory effects of ignored primes. Quarterly Journal of Experimental Psychology, 37, $571-590$.

Vanderplas, J. M., \& Garvin, E. A. (1959). The association value of random shapes. Journal of Experimental Psychology, 57, 147-154.

Veling, H., Holland, R. W., \& van Knippenberg, A. (2007). Devaluation of distracting stimuli. Cognition \& Emotion, 21, 442-448.

Vuilleumier, P., Schwartz, S., Duhoux, S., Dolan, R. J., \& Driver, J. (2005). Selective attention modulates neural substrates of repetition priming and "implicit" visual memory: Suppressions and enhancements revealed by fMRI. Journal of Cognitive Neuroscience, $17,1245-1260$.

Whittlesea, B. W. A. (1993). Illusions of familiarity. Journal of 
Experimental Psychology: Learning, Memory, and Cognition, 19, $1235-1253$.

Whittlesea, B. W. A., \& Price, J. R. (2001). Implicit/explicit memory versus analytic/nonanalytic processing: Rethinking the mere exposure effect. Memory \& Cognition, 29, 234-246.

Willems, S., \& Van der Linden, M. (2006). Mere exposure effect: A consequence of direct and indirect fluency-preference links. Consciousness and Cognition, 15, 323-341.

Wilson, W. R. (1979). Feeling more than we can know: Exposure effects without learning. Journal of Personality and Social Psychology, 37, 811-821.

Winkielman, P., \& Cacioppo, J. T. (2001). Mind at ease puts a smile on the face: Psychophysiological evidence that processing facilitation elicits positive affect. Journal of Personality \& Social Psychology, 81, 989-1000.

Zajonc, R. B. (1968). Attitudinal effects of mere exposure. Journal of Personality and Social Psychology, Monograph Supplement, 9, 1-27. 


\section{Author Note}

\section{Yoshihiko Yagi}

National Institute of Advanced Industrial Science and Technology

Shinobu Ikoma, and Tadashi Kikuchi

University of Tsukuba, Graduate School of Comprehensive Human

Sciences.

This research was supported in part by the following grants from the Japan Society for the Promotion of Science (JSPS): "Promotion of Kansei science for understanding mind mechanism" of the University of Tsukuba based on the COE program for the 21st century; a Grant-in-Aid for Scientific Research (C) No.20530657; and Research Fellowships of the JSPS for Young Scientists for Y.Y.

Correspondence concerning this article should be addressed to Yoshihiko Yagi, National Institute of Advanced Industrial Science and Technology (AIST), 1-1-1 Higashi, Tsukuba, 305-8566 Ibaraki Japan E-mail:y-yagi@aist.go.jp Phone: +81 29-861-6724(lab.) 
Table 1

Mean Selection Rates for Exposed Drawings (SE) in Affective Judgment Phase and Mean Proportion Correct Recognition (SE) in Recognition Phase in Each Experiment

\begin{tabular}{lcccc}
\hline & $\begin{array}{c}\text { To-be-attended } \\
\text { drawings }\end{array}$ & $\begin{array}{c}\text { To-be-evaluated } \\
\text { drawings }\end{array}$ & $\begin{array}{c}\text { Proportion exposed } \\
\text { drawings selected }\end{array}$ & $\begin{array}{c}\text { Proportion of correct } \\
\text { recognition }\end{array}$ \\
\hline $\begin{array}{c}\text { Exp. 1 } \\
\text { Attended } \\
\text { Ignored }\end{array}$ & Components & Components & $.55(0.01)$ & $.63(0.02)$ \\
Exp. 2 & & & $.48(0.02)$ & $.51(0.01)$ \\
Attended & Components & Composites & $.49(0.02)$ & $.69(0.02)$ \\
Ignored & Composites & Components & $.58(0.02)$ & $.52(0.01)$ \\
Exp. 3 & Composites & Components & $.50(0.01)$ & $.60(0.02)$ \\
Exp. 4a & Com & $.51(0.01)$ & $.54(0.01)$ \\
Exp. 4b & Composites & Components & $.51)$ \\
\hline
\end{tabular}


Drawings presented in the exposure phase

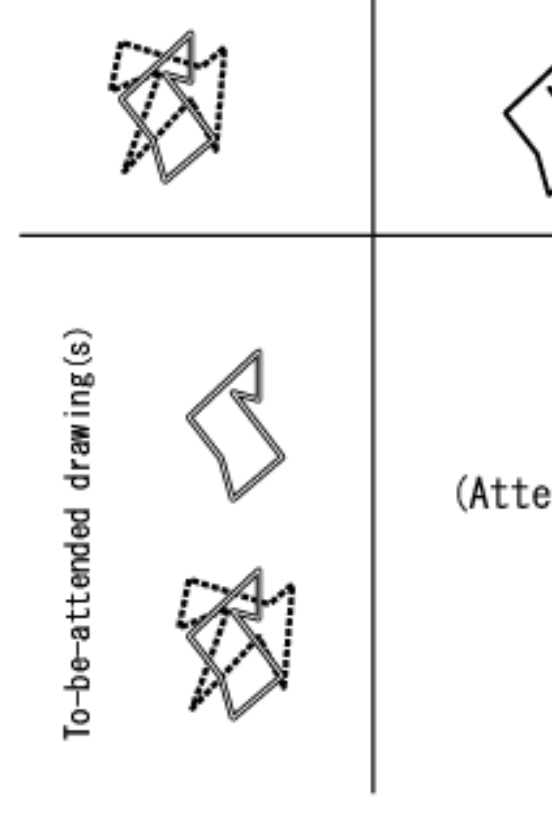

To-be-evaluated drawing (s) in the affective decision phase

Exp. 1

Exp. 2

Exp. 3

Figure 1 


\section{Figure Caption}

Figure 1. The relationship between the to-be-attended drawing(s) in the exposure phase and the to-be-evaluated drawing(s) in the affective judgment phase in each experiment. 